\title{
Fingertip amputation injury managed conservatively with moist wound dressings
}

\author{
Shigenori Masaki ${ }^{1}$ and Takashi Kawamoto ${ }^{1}$ \\ ${ }^{1}$ Miyanomori Memorial Hospital
}

August 12, 2020

\begin{abstract}
A 36-year-old woman suffered an Allen type III fingertip amputation of the right third finger. The wound was treated conservatively using moist wound dressings and healed after 12 weeks with excellent esthetic and functional results. Conservative management using moist wound dressings can be successful treatment for fingertip amputation injury.
\end{abstract}

\section{Introduction}

Fingertip amputation injuries are commonly encountered in emergency departments. Management of these injuries varies depending on the location and extent of tissue loss. A classification system for fingertip amputations was described by Allen. ${ }^{1}$ Fingertip amputations of Allen type III/IV tend to be treated surgically. ${ }^{2}$ However, conservative management may be a viable treatment option. ${ }^{3}$ A particular management strategy for these types of injuries has not been finalized. Herein, we report a case of an Allen type III fingertip amputation that was managed conservatively with moist wound dressings.

\section{Case report}

A 36-year-old woman crushed the third finger of her right hand in a thick iron door and suffered a fingertip amputation injury. She was a smoker and her medical history was unremarkable. She initially visited the emergency department at another general hospital. The amputated part of the finger was not collected. The plastic surgeon handling the case recommended surgical reconstruction. However, the patient wished to receive conservative management and visited the wound care department in our hospital. During the initial visit, it was noted that the nail plate of the finger was lost, the nail bed was injured, and the distal phalanx of the finger was exposed, indicating that her fingertip amputation was an Allen type III (Figure 1 A, B). Conservative management was provided using Plus moist ${ }^{\mathrm{TM}}$ (ZUIKO MEDICAL, Osaka, Japan), which is a multi-layered moist wound dressing made of polyethylene, polypropylene, and cellulose (Figure 2). ${ }^{4}$ Informed written consent was obtained from the patient for the publication of this case report and any accompanying images. Ethical approval was obtained from the Ethical Review Board of Miyanomori Memorial Hospital.

\section{Outcome and follow-up}

After initiation of conservative management with Plus moist ${ }^{\mathrm{TM}}$, the patient returned for evaluation and follow-up once every 1-2 weeks. The protocol of this management strategy was to cover the wound with Plus moist ${ }^{\mathrm{TM}}$. The patient changed the dressing at home once per day after gently washing the wound with hot water in the shower. Disinfectants and prophylactic antibiotics were not used in this case based on the criteria of our wound care department. Two weeks later, we confirmed the generation of a new nail plate and granulation of the finger (Figure $1 \mathrm{C}$ ). Four weeks later, granulation increased, and the tip regained a shape similar to that of the original finger (Figure 1 D). Eight weeks later, epithelialization neared completion, but there was slight granulation at the tip (Figure $1 \mathrm{E}$ ). Twelve weeks later, epithelialization was 
completed (Figure $1 \mathrm{~F}$ ). Follow-up was decreased to once every 4 weeks. The patient initially complained of mild pain when the fingertip was touched, however, the pain gradually subsided. The patient complained of fingertip numbness at 16 weeks and slight cold intolerance at 27 weeks, but these also gradually improved. To address discomfort, pregabalin $150 \mathrm{mg} /$ day, mecobalamin $1500 \mu \mathrm{g} /$ day, and loxoprofen sodium hydrate $180 \mathrm{mg} /$ day were administered separately or simultaneously as needed. During about the first 6 months after the injury, the patient occasionally had a little difficulty with holding a pen and chopsticks due to the pain and numbness, but gradually recovered her ability to function. Local and systemic infections did not occur. A slight shortening of the fingertip was observed at the end of follow-up at 39 weeks (Figure 3). At the time of the final follow-up, the patient was able to live the same daily life as before the injury. She was satisfied with the overall treatment result.

\section{Discussion}

We present a case of an Allen type III fingertip amputation, managed conservatively with moist wound dressings. The wound epithelialized at 12 weeks. The esthetic and functional results were excellent. There are two management strategies for fingertip amputations: operative and conservative management. Management strategies vary by country and region. Conservative management of fingertip amputations is popular in the United States and European countries. ${ }^{5,6}$ However, operative management is preferred over conservative in Asian countries. ${ }^{5,7}$ In Japan, replantation and reconstruction are the major strategies for fingertip amputations $;{ }^{8-10}$ however, conservative management using moist wound dressings has become more common in recent years. ${ }^{4}$ In the current case, a plastic surgeon recommended surgery during the initial visit; however, the patient chose conservative management because, after thoroughly reviewing medical articles regarding the treatment of fingertip injuries, she believed that conservative management would bring better outcomes rather than surgery.

Conservative management for fingertip amputations is generally performed using moist wound dressings. ${ }^{11}$ Various types of dressings such as self-adhesive elastic bandages with Vaseline, ${ }^{11}$ UrgoTul, ${ }^{12}$ and film dressings ${ }^{13}$ can be used. In this case, Plus moist ${ }^{\mathrm{TM}}$, a popular moist wound dressing in Japan, was used. The characteristics of Plus moist ${ }^{\mathrm{TM}}$ include its non-adherent ability and self-regulating absorption of the exudate. ${ }^{4}$ Patients can buy Plus moist ${ }^{\mathrm{TM}}$ from drugstores or online stores, cover the wound and change the dressing once per day. Therefore, Plus moist ${ }^{\mathrm{TM}}$ is easy to handle and is widely accepted among patients. ${ }^{4}$ However, since the available wound dressings differ depending on the country or region, the most suitable wound dressing for treating fingertip amputations has not been verified. Therefore, individual hospitals should select dressings that are appropriate and accessible for their facility.

Regarding time to healing, operative management, especially in revision tip amputations, provides faster healing than conservative management. ${ }^{3,5}$ Although time to healing in conservative management depends on the size of the defects, the mean healing time has been reported as $2-12$ weeks. ${ }^{11}$ Fingertip amputations with exposed bone require the longest time to heal. ${ }^{14,15}$ In this case, it took 12 weeks to completely heal. The size of the defect and the exposed bone may have been associated with a relatively long healing time. Furthermore, the patient's smoking may have affected the wound healing time. ${ }^{5,16}$

In reported studies of amputation with exposed bone, surgical shortening of the bone was often recommended to facilitate healing. ${ }^{11,17}$ Conversely, other studies indicated that the protruding bone should not be removed unless there are sharp bone spicules present because bone trimming results in the loss of the bony support of the fingertip and increases the possibility of a hook nail deformity. ${ }^{15}$ In this case, trimming of the exposed bone was deemed unnecessary and was not performed. As a result, no nail deformity was observed. The soft tissue thickness has been reported to regenerate up to $85 \%$ and $93 \%$ in the palmar and distal directions, respectively, compared to that on the unaffected side. ${ }^{18}$ The thickness of the regenerated fingertip in this case was the same as that reported in previous studies. Interestingly, as granulation gradually increased, the tip regained a shape similar to that of the original finger at around 4 weeks. Furthermore, the nail bed regenerated normally, showing excellent esthetic results.

Regarding sensation, cold intolerance is a common problem which frequently improves over time and resolves 
by 1 year. ${ }^{11}$ Cold intolerance is not specific to conservative management and is also common in operative management. ${ }^{5,11,14}$ In this case, the patient complained of cold intolerance at 27 weeks, just at the beginning of winter. It is thought that cold intolerance became evident due to the decrease in temperature. Changes in sensitivity are seen especially in proximal injuries, which can occasionally be considered an unsatisfactory result for the patients. ${ }^{1,5,18}$ Also, in this case, numbness adversely affected the patient's daily activities. However, both cold intolerance and numbness improved over time and resolved. It is important to inform patients in advance that sensory abnormalities can occur and continue for a while but frequently improve over time, which may lead to improvement in patient acceptance of this management strategy.

Although conservative management of fingertip amputations may require a longer healing time, there are several advantages of conservative management over operative management: good esthetic and functional results with near-normal sensitivity, no need for admission and immobilization, and no post-surgery complications. Therefore, conservative management can be more effective than operative management. ${ }^{11,14}$ When conservative management is an option for a fingertip amputation, it is recommended that doctors should fully explain the advantages and disadvantages of operative and conservative management to the patients in advance, and select the course of treatment keeping the patient's wishes as the first priority.

\section{Conclusion}

Conservative management using moist wound dressings for fingertip amputation injury is simple and overall treatment results can be satisfactory for the patient. Therefore, conservative management can be considered as a preferable management strategy for fingertip amputation injury depending on the nature and extent of the injury.

\section{Acknowledgements}

There was no financial support for this work.

We would like to thank Editage (www.editage.jp) for English language editing.

\section{Conflict of Interest}

None declared.

\section{Author contribution}

Masaki S: involved in the conception and design of this case report, acquisition and interpretation of data, drafting of the manuscript, critical revision of the manuscript for important intellectual content, and final approval of the manuscript.

Kawamoto T: involved in the conception and design of this case report, critical revision of the manuscript for important intellectual content, and final approval of the manuscript.

\section{References}

1. Allen MJ. Conservative management of finger tip injuries in adults.Hand. 1980;12:257-265. doi: 10.1016/S0072-968X(80)80049-0

2. Arsalan-Werner A, Brui N, Mehling I, Schlageter M, Sauerbier M. Long-term outcome of fingertip reconstruction with the homodigital neurovascular island flap. Arch Orthop Trauma Surg.2019;139:1171-1178. doi: 10.1007/s00402-019-03198-4

3. Germann G, Rudolf KD, Levin SL, Hrabowski M. Fingertip and thumb tip wounds: Changing algorithms for sensation, aesthetics, and function.J Hand Surg Am . 2017;42:274-284. doi: 10.1016/j.jhsa.2017.01.022

4. Natsui M. Overview of wound healing in a moist environment 2012.http://www.woundtreatment.jp/english/index_e.htm. Accessed June 22, 2020.

5. Sindhu K, DeFroda SF, Harris AP, Gil JA. Management of partial fingertip amputation in adults: Operative and non operative treatment.Injury . 2017;48:2643-2649. doi: 10.1016/j.injury.2017.10.042 
6. Elliot D, Adani R, Hyun Woo S, Tang JB. Repair of soft tissue defects in finger, thumb and forearm: less invasive methods with similar outcomes. J Hand Surg Eur Vol . 2018;43:1019-1029. doi: $10.1177 / 1753193418805698$

7. Karjalainen T, Sebastin SJ, Chee KG, Peng YP, Chong AKS. Flap related complications requiring secondary surgery in a series of 851 local flaps used for fingertip reconstruction. $J$ Hand Surg Asian Pac Vol . 2019;24:24-29. doi: 10.1142/S242483551950005X

8. Koshima I, Yoshida S, Imai H, et al. Recent topics on fingertip replantations under digital block. Hand Clin . 2019;35:179-184. doi: 10.1016/j.hcl.2018.12.007

9. Usami S, Okazaki M. Fingertip reconstruction with a posterior interosseous artery perforator flap: A minimally invasive procedure for donor and recipient sites. J Plast Reconstr Aesthet Surg . 2017;70:166-172. doi: $10.1016 /$ j.bjps.2016.11.027

10. Osada A, Matsumine H, Kamei W, Sakurai H. Usefulness of avulsed fingertip skin for reconstruction after digital amputation. Case Reports Plast Surg Hand Surg . 2020;7:23-29. doi: 10.1080/23320885.2020.1719843

11. Krauss EM, Lalonde DH. Secondary healing of fingertip amputations: a review. Hand . 2014;9:282-288. doi: $10.1007 / \mathrm{s} 11552-014-9663-5$

12. Ng HJH, Sim J, Tey VHT, Selvaganesh S, Rebosura CKP, Rajaratnam V. Experience with the use of splint caps for the management of fingertip amputation injuries. J Hand Surg Asian Pac Vol . 2020;25:199205. doi: 10.1142/S242483552050023X

13. Kurian S, Davis M, Fazi A, McClellan WT. IV 3000 dressing for fingertip injury: management and discussion. WV Med J . 2016;112:24-26.

14. Kawaiah A, Thakur M, Garg S, Kawasmi SH, Hassan A. Fingertip injuries and amputations: a review of the literature. Cureus . 2020;12:e8291. doi: 10.7759/cureus.8291

15. Champagne L, Hustedt JW, Walker R, Wiebelhaus J, Nystrom NA. Digital tip amputations from the perspective of the nail. Adv Orthop . 2016;2016:1967192. doi: 10.1155/2016/1967192

16. Álvarez-Jiménez J, Córdoba-Fernández A. Influence of smoking on wound healing in patients undergoing nail matrix phenolization: a prospective randomized clinical study. Adv Skin Wound Care . 2014;27:229-236. doi: 10.1097/01.ASW.0000444612.93346.6c

17. Weichman KE, Wilson SC, Samra F, Reavey P, Sharma S, Haddock NT. Treatment and outcomes of fingertip injuries at a large metropolitan public hospital. Plast Reconstr Surg . 2013;131:107-112. doi: 10.1097/PRS.0b013e3182729ec2

18. Hoigné D, Hug U, Schürch M, Meoli M, von Wartburg U. Semi-occlusive dressing for the treatment of fingertip amputations with exposed bone: quantity and quality of soft-tissue regeneration. J Hand Surg Eur Vol . 2014;39:505-509. doi: 10.1177/1753193413489639

\section{Figure legends}

Figure 1

Initial presentation of fingertip amputation injury on the third finger of the right hand. The distal phalanx of the finger is exposed during the initial visit (A, B). Granulation increases at 2 weeks $(\mathrm{C})$. The tip regains a shape similar to that of the original finger at 4 weeks (D). Epithelialization near completion at 8 weeks (E). Complete epithelialization at 12 weeks (F).

Figure 2

Plus moist ${ }^{\mathrm{TM}}$ consists of three layers. The outer side is a leak-proof layer that prevents the exudate from leaking (A), the middle part is an absorbent layer that absorbs the exudate (B), and the inner side is a 
permeable layer that comes in contact with the wound $(\mathrm{C})$. Patients cover the wound with Plus moist ${ }^{\mathrm{TM}}$ and change it once per day (D).

Figure 3

Fingertip on the palmar and dorsal surfaces at 39 weeks (A, B). Range of motion of the fingertip is normal (C, D).
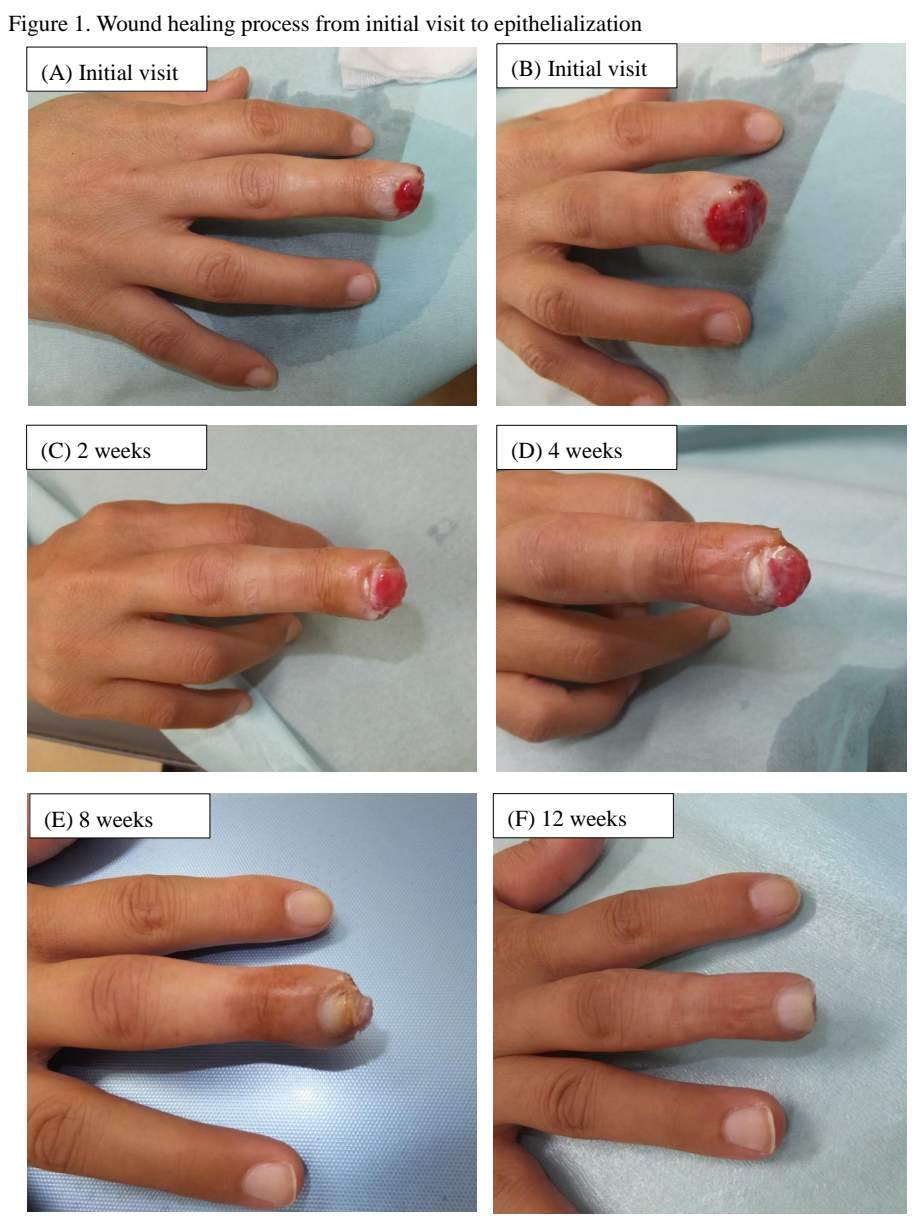
Figure 2. The moist wound dressing (Plus moist ${ }^{\mathrm{TM}}$ ) used in this case
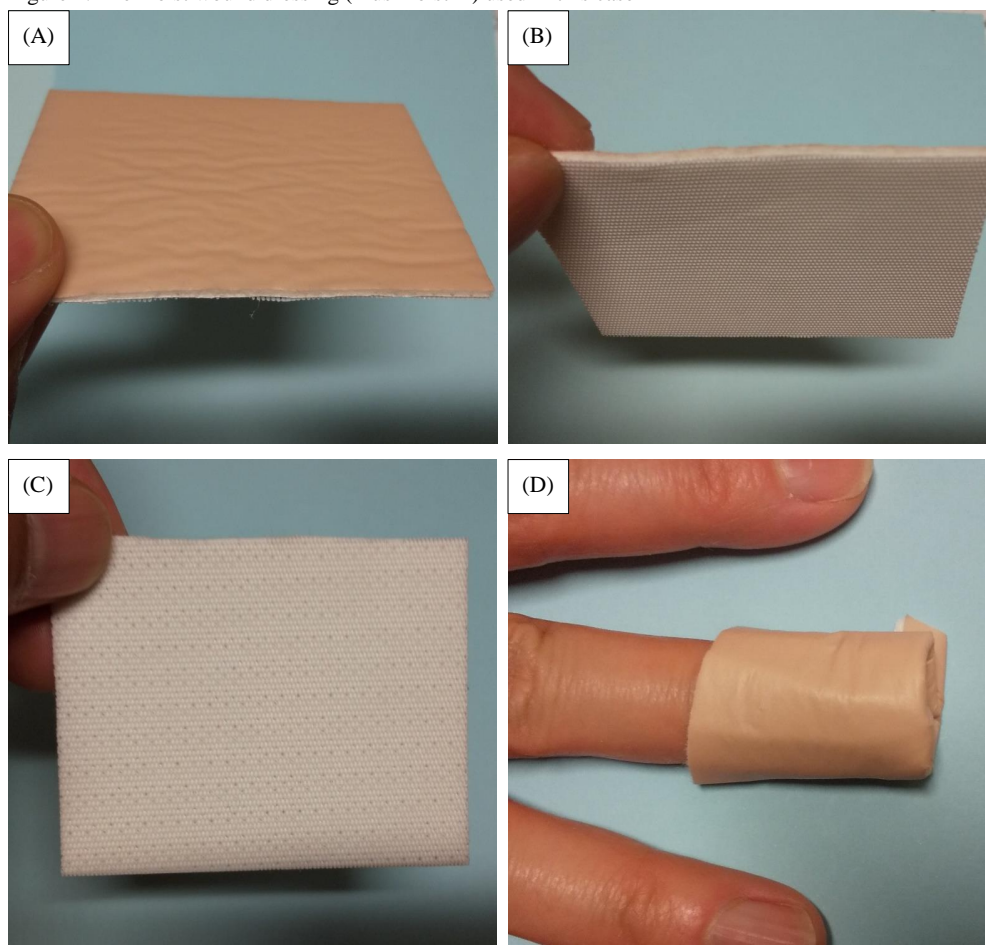
Figure 3. Appearance and range of motion after 39 weeks

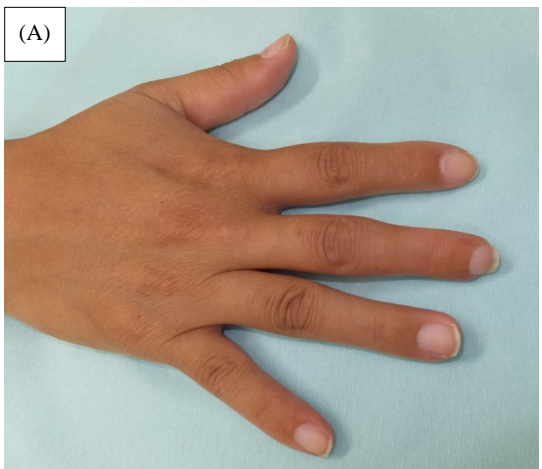

(B)
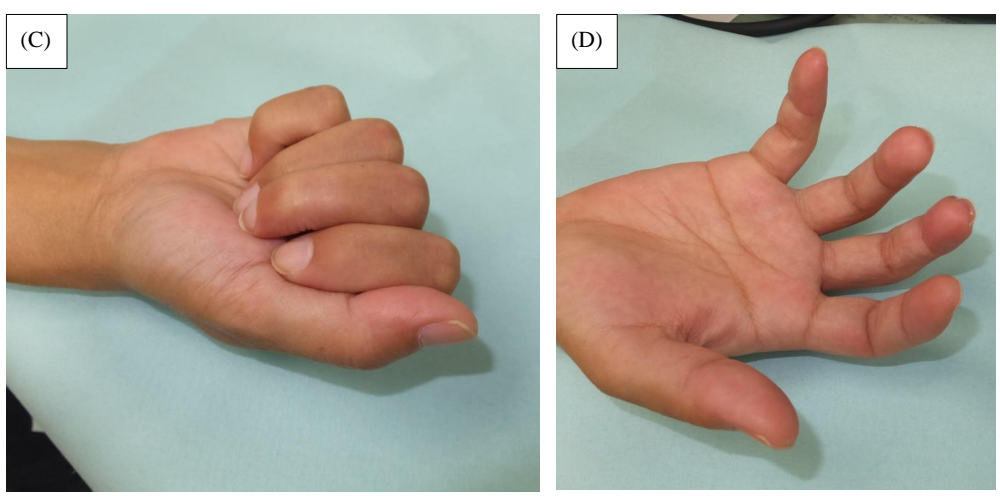